\section{If We Build It, They Will Come: Rethinking Some Assumptions About Screening and Intervening for Distress}

\author{
Steven C. Palmer, PhD
}

I $\mathrm{n}$ this issue of JNCCN, Schuurhuizen et al ${ }^{1}$ provide an opportunity to examine some assumptions about screening and intervening for psychosocial distress among patients with cancer. In their study, 349 individuals with metastatic colorectal cancer were cluster randomized by hospital to either a stepped care intervention for distress or usual care. Although "elevated" distress was more prevalent than expected (at least partly due to a low cutpoint on the Hospital Anxiety and Depression Scale ${ }^{2}$ to improve sensitivity), so few individuals in the stepped care arm exposed themselves to active intervention that a futility analysis was performed. The study stopped recruitment when it was determined that $>33,000$ participants would be needed to give the study adequate power to detect the observed effect size. Results of this study, in conjunction with a relative lack of high-quality evidence for routine approaches to distress intervention, ${ }^{3,4}$ add more weight to the need to examine current practices and assumptions. In particular, it may be worthwhile to examine how screening for distress differs from biomedical screening and intervention, how the construction and context of distress might complicate efforts to improve patient outcomes, and why the assumption that "if we build it, they will come" appears to be less the case than was anticipated.

Screening and intervening for distress differs from biomedical screening in a number of ways. Screening, in general, focuses on detecting relatively nonsymptomatic conditions that would otherwise not be identified but require effective intervention (eg, hypertension). Distress, however, does not align with this model. Unlike biomedical screening, distress screening rests on the assumption that patients recognize their distress, can report it and its sources, and are willing to do so. However, most approaches to distress screening are "top-down," ${ }^{5}$ relying on patients meeting a predetermined threshold that prompts further assessment and intervention.

In essence, these approaches prioritize expert opinion as to what distress means, what level requires intervention, and usually what that the intervention consists of. Uptake of interventions among screened patients is often modest, at best $^{6}$ and the usual approaches to screening are likely to identify many individuals as distressed who are unlikely to accept psychosocial intervention.

An unintended consequence of top-down approaches to distress screening is that they not only overidentify individuals as distressed who will ultimately reject intervention but also can prevent individuals who desire intervention from obtaining it simply because they fail to meet some arbitrary cutpoint. Data are now starting to emerge in other populations showing that these "non-distressed" but help-desiring individuals make up a large proportion of those who desire intervention. ${ }^{7,8}$ This has sparked interest in "bottom-up" approaches and hybrid models 4 that rely more on structured patient reports of unmet needs and their desire for intervention. These models potentially streamline triage to intervention and allow individuals who do not meet screening criteria to obtain the intervention they want. Schuurhuizen et al ${ }^{1}$ included expressed "need for psychosocial care" as a possible criterion to trigger intervention, which is a potential improvement but assumes that the participant wants a psychosocial intervention rather than some other type of care.

There are several potential reasons why patients may not avail themselves of services offered to reduce their distress; one that is often neglected that patients may not

See page 911 for related article.

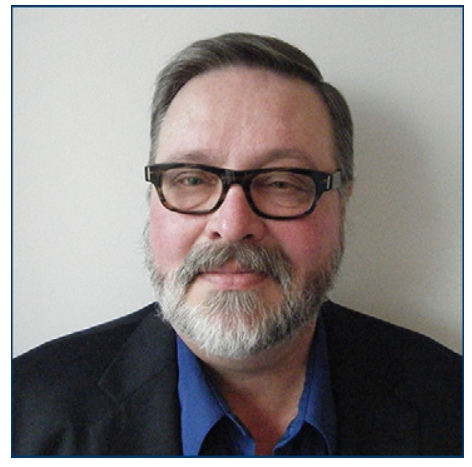

STEVEN C. PALMER, PhD

Steven C. Palmer, PhD, is a Clinical Health Psychologist and Senior Research Investigator of Clinical Research Studies in the Survivorship Program at the Abramson Cancer Center at the University of Pennsylvania. His research interests range from prediction and remediation of psychosocial difficulties and chronic distress to supporting the transition from acute to long-term medical care among cancer survivors.

doi: $10.6004 /$ jnccn.2019.7340

The ideas and viewpoints expressed in this commentary are those of the author and do not necessarily represent any policy, position, or program of NCCN 
see the intervention as relevant to the source of the distress. Distress is not a unitary construct but a "multifactorial unpleasant experience of a psychological, social, spiritual, and/or physical nature that may interfere with the ability to cope effectively with cancer, its physical symptoms, and its treatment." 9 Distress often arises from nonpsychological issues. Both the NCCN Guidelines for Distress ${ }^{9}$ and the design of the Distress Thermometer (DT) recognize this through the inclusion of the Problem List $(\mathrm{PL}), \mathrm{a}$ checklist of 39 potential sources of distress, which include practical, emotional, family, spiritual, and physical problems.

Distress screening and intervention, however, often focus not on identifying and alleviating the problems individuals are experiencing and that are reflected in distress scores, but rather on reducing psychosocial distress itself, as though it is a more unitary construct amenable to a limited number of effective interventions. In essence, top-down approaches risk neglecting the understanding that the "map is not the territory"10 and mistaking our metric of distress for distress itself. Thus, if we focus attention on the distress score, we risk not considering the perceived sources of distress. This may be particularly vexing, given that distress is both multifactorial and multiply determined. One size, or even a series of steps of one size, is unlikely to fit all.

In Schuurhuizen et al, for example, although the DT/PL were administered along with other instruments, the intervention offered stepped care, moving from watchful waiting, to self-help, to face-to-face problem-solving, to referral regardless of the problems participants were experiencing. This stepped care may make sense if the intervention target is distress itself, but it may seem irrelevant to a patient experiencing distress due to issues like pain, financial strain, or lack of transportation-instrumental interventions that might improve patient outcomes (eg, improving pain treatment, immediate referral to social work, or arranging transportation) are superseded by psychosocial interventions that target the distress. This could certainly impair uptake of services.

Similarly, context matters when offering interventions and expecting uptake. One contextual factor that appears under-addressed in the literature is that of the patient population and how other factors might influence their needs and abilities to engage in intervention. In Schuurhuizen et al, patients had metastatic colorectal cancer with a life expectancy of $>3$ months - a particularly vulnerable population. More than $20 \%$ of patients died during the study period, which was more than those who exposed themselves to active intervention. In the stepped care intervention arm, 47 patients died and only 21 accepted any active treatment, despite 111 screening positive for distress at some point. With a mortality rate this high, one must wonder whether participants had different needs than the investigators anticipated. It may simply be that a psychosocial approach to their distress and its sources did not seem particularly relevant given the context of their advanced disease.

What if we build it and they do not come? Schuurhuizen et al present a well-designed and well-reported study that found uptake of an offered intervention for distress so low as to render the study infeasible. When offered a stepped care intervention, patients voted with their feet and declined the offer. Although the reason is not clear, it seems plausible that they did not perceive the intervention as relevant to their needs, abilities, and priorities, particularly in the context of a life-threatening illness. When considered with other studies producing similar results, this study may suggest a need to rethink top-down approaches to distress screening and intervention in favor of more bottom-up approaches that better reflect the needs and priorities of patients.

One approach may be to inquire upfront about patient desire for intervention with their specific problems and to address these as a means of improving outcomes; that is, focusing on what patients want assistance with in a manner that is consistent with their perceived needs and abilities. Studies have recently found that although most patients who report elevated distress do not desire intervention within the cancer care setting, a substantial proportion of those who do desire intervention do not screen positive for distress and will likely be missed unless patient-perceived need for intervention is assessed. ${ }^{7,8}$ Not prioritizing the patient perspective when detecting and treating the difficulties that underlie distress risks overidentifying need for intervention while missing the opportunity to improve outcomes for a large number of individuals who desire intervention.

\section{References}

1. Schuurhuizen CSEW, Braamse AMJ, Beekman ATF, et al. Screening and stepped care targeting psychological distress in patients with metastatic colorectal cancer: the TES cluster randomized trial. J Natl Compr Canc Netw 2019;17:911-920

2. Zigmond AS, Snaith RP. The hospital anxiety and depression scale. Acta Psychiatr Scand 1983;67:361-370

3. McCarter $\mathrm{K}$, Britton $\mathrm{B}$, Baker $\mathrm{AL}$, et al. Interventions to improve screening and appropriate referral of patients with cancer for psychosocial distress: systematic review [published online January 5, 2018]. BMJ Open, doi: 10.1136/bmjopen-2017-017959

4. Meijer A, Roseman M, Delisle VC, et al. Effects of screening for psychological distress on patient outcomes in cancer. J Psychosom Res 2013;75:1-7.

5. Sanson-Fisher R, Carey M, Paul C. Measuring the unmet needs of those with cancer. Cancer Forum 2009;33:198-201.

6. Brebach R, Sharpe L, Costa DS, et al. Psychological intervention targeting distress for cancer patients. Psychooncology 2016;25:882-890.

7. Philip EJ, Merluzzi TV. Psychosocial issues in post-treatment cancer survivors: desire for support and challenges in identifying individuals in need. J Psychosoc Oncol 2016;34:223-239

8. Jacobs LA, Blauch AN, Pucci DA, et al. Does screening for anxiety and depression efficiently identify patients who want help? J Clin Oncol 2014 35(Suppl):e21604.

9. Riba MB, Donovan KA, Anderson B, et al. NCCN Clinical Practice Guidelines in Oncology: Distress Management. Version 3.2019. To view the most recent version, visit NCCN.org. Accessed July 7, 2019.

10. Korzybski A. Science and Sanity. An Introduction to Non-Aristotelian Systems and General Semantics. Oxford, England: Institute of General Semantics; 1933. 